P117 DISSEMINATED GONOCOCCAL INFECTION CASE SERIES: CLINICAL PRESENTATIONS, MICROBIOLOGY FINDINGS AND WHOLE GENOME SEQUENCING RESULTS

${ }^{1} \mathrm{~J}$ Murira*, ${ }^{1} \mathrm{~K}$ Phyu, ${ }^{1} \mathrm{~W}$ Fawley, ${ }^{1} \mathrm{~J}$ Carter, ${ }^{2} \mathrm{~A}$ Walker, ${ }^{3} \mathrm{D}$ Eyre, ${ }^{1} \mathrm{M}$ Wilcox, ${ }^{1} \mathrm{~J}$ Wilson. ${ }^{1}$ Leeds Teaching Hospitals Trust, Leeds, UK; ${ }^{2}$ Nuffield Department of Medicine, University of Oxford, Oxford, UK; ${ }^{3}$ Big Data institute, University of Oxford, Oxford, UK

\subsection{6/sextrans-2021-sti.238}

Background Disseminated gonococcal infection (DGI) is a rare complication of Neisseria gonorrhoeae (NG) infection arising from bacteraemia. It classically manifests as one or more of acute arthritis, tenosynovitis and dermatitis, more commonly in females. Anecdotally, DGI is increasing. We describe eight cases of DGI; seven presented within 13 months.

Methods All cases presented/referred, to STI/ID at a large teaching hospital, in England and diagnosed with proven/probable DGI.

Demographic, clinical and microbiological data were prospectively collected.

Whole genome sequencing (WGS) of positive cultures was undertaken.

Results Three proven (synovial fluid/skin NG culture positive) and five probable cases were identified, all males (7/8 MSM), and most (7/8) with no genital symptoms. Hip and wrist were the most commonly affected joints. Dermatitis featured in $5 / 8$. All were NG NAAT-positive at an extra-genital site (pharynx or rectum); only $3 / 8$ were urine NAAT-positive. Five cultured isolates from five patients underwent WGS: $2 / 5$ had no linkage to other DGI or database isolates, $3 / 5$ were $8-20$ single nucleotide polymorphisms apart and from ST-7822, one of commonly circulating lineages in England. 6/8 presented initially to non-STI specialties, and experienced a delay in diagnosis and initiation of appropriate antibiotic therapy (mean 10.7 versus 4 days). Five required hospital admission (mean stay 14.4 days).

Conclusion This is the first study of WGS in DGI. DGI cases were not all associated with a single clone or outbreak, but several were from the same lineage, consistent with ST-7822 possibly causing more invasive infection. Our series supports an increased rate of DGI (our department saw $<1$ case/year historically). This may be related to host factors combined with increased NG prevalence in MSM worldwide. The cases demonstrate the lack of genital symptoms, which may contribute to delay in management and highlights the importance of extra-genital NAAT testing and awareness outside of STI services.

\section{P119 COMMUNITY-DIRECTED BACTERIAL SEXUALLY TRANSMITTED INFECTION (STI) TESTING INTERVENTIONS AMONG MEN WHO HAVE SEX WITH MEN (MSM): AN E-DELPHI STUDY}

\footnotetext{
${ }^{1}$ A Yeung ${ }^{*},{ }^{2} \mathrm{R}$ Lisk, ${ }^{1,3} \mathrm{~J}$ Rana, ${ }^{1,3,4,5} \mathrm{C}$ Guiang, ${ }^{5} \mathrm{~J}$ Bacon, ${ }^{6} \mathrm{~J}$ Brunetta, ${ }^{7} \mathrm{M}$ Gilbert, ${ }^{3} \mathrm{D}$ Gesink, ${ }^{1,3} \mathrm{R}$ Grewal, ${ }^{8} \mathrm{M}$ Kwag, ${ }^{3} \mathrm{C}$ Logie, ${ }^{4} \mathrm{~L}$ Mitterni, ${ }^{9} \mathrm{R}$ Shahin, ${ }^{1,3} \mathrm{D}$ Tan, ${ }^{1,3} \mathrm{~A}$ Burchell. ${ }^{1}$ Unity Health Toronto, Toronto, Canada; ${ }^{2}$ ACT, Toronto, Canada; ${ }^{3}$ University of Toronto, Toronto, Canada; ${ }^{4}$ Hassle Free Clinic, Toronto, Canada; ${ }^{5}$ Ontario HIV Treatment Network, Toronto, Canada; ${ }^{6}$ Maple Leaf Medical Clinic, Toronto, Canada; ${ }^{7} B C$ Centre for Disease Control, Vancouver, Canada; ${ }^{8}$ Community-Based Research Centre, Vancouver, Canada; ${ }^{9}$ Toronto Public Health, Toronto, Canada
}

10.1136/sextrans-2021-sti.239
Background Canadian clinical guidelines recommend at least annual and quarterly sexually transmitted infection (STI) testing among sexually active men who have sex with men (MSM), including those on HIV PrEP or in HIV care. We built consensus around interventions to improve local STI testing services for MSM using a web-based 'e-Delphi' process. Methods We recruited Experts for a Community Panel (MSM who sought/underwent STI testing in the preceding 18 months, conducted 09/2019-11/2019) and a Provider Panel (offered STI testing to MSM in the past 12 months, conducted 02/2020-05/2020). Experts prioritized 6-8 potential interventions, generated from a literature review, on a 7-point Likert scale of 'Definitely not a priority' to 'Definitely a priority' over 3 survey rounds. Consensus was defined as $\geq 60 \%$ within a \pm 1 response point. Summaries of panel responses were given in successive rounds. We report the percentage of 'a priority' at the final round of survey.

Results Among Community Experts, 43/51 (84\%) completed all rounds; 19\% HIV-positive, 37\% HIV-negative on PrEP, 42\% HIV-negative not on PrEP. We reached consensus on 6 interventions - Client Reminders (95\%), Express testing (88\%), Routine testing (84\%), Online booking app (84\%), Online testing (77\%) and Nurse-led testing (72\%). Experts favoured interventions that were convenient while also maintaining a relationship with their provider. Among Provider Experts, 37/48 (77\%) completed all rounds; 59\% were physicians. Consensus was reached on the aforementioned interventions (range 68\%-100\%) but not reached for Provider Alerts (19\%) and Provider Audit and Feedback (16\%). Express, Online and Nurse-led testing were prioritized by $>95 \%$ of Experts because of streamlined processes and decreased need to see a provider.

Conclusions Both panels were enthusiastic about innovations that make STI testing more efficient. However, Community Experts preferred convenient interventions that involved their provider while Provider Experts favoured interventions that prioritized patient independence and reduced patient-provider time.

\section{P120 INCARCERATION AND SUBSEQUENT PREGNANCY LOSS AMONG BLACK AND WHITE WOMEN: EXPLORATION OF SEXUALLY TRANSMITTED INFECTIONS AS A MEDIATING PATHWAY}

'J Scheidell*, ${ }^{2} \mathrm{~T}$ Dyer, ${ }^{3} \mathrm{~A}$ Knittel, ${ }^{1} \mathrm{E}$ Caniglia, ${ }^{1} \mathrm{~L}$ Thorpe, ${ }^{1} \mathrm{~A}$ Troxel, ${ }^{4} \mathrm{C}$ Lejuez, ${ }^{1} \mathrm{M}$ Khan. ${ }^{1}$ NYU Grossman School of Medicine, New York, USA; ' University of Maryland School of Public Health, College Park, USA; ' University of North Carolina at Chapel Hill, Chapel Hill, USA; ${ }^{4}$ University of Connecticut, Storrs, USA

\subsection{6/sextrans-2021-sti.240}

Background Incarceration is linked to risk of sexually transmitted infection (STI) post-release among women in the United States. There has been little examination of incarceration's association with related sexual and reproductive outcomes such as pelvic inflammatory disease (PID) and pregnancy loss, or the role of STI in this relationship and whether these relationships differ between Black and white women.

Methods Using data from the National Longitudinal Study of Adolescent to Adult Health, we examined cross-sectional associations between incarceration (Wave IV; 2007-08; ages 24-34) and history of STI and PID ( $n=5968)$, and 
longitudinal associations between incarceration and later pregnancy loss in mid-adulthood (Wave V; 2016-18; ages 34-43) among women who had ever been pregnant $(n=2353)$; we estimated racial differences. Using causal mediation, we explored whether STI and PID mediated associations with pregnancy loss.

Results Incarceration was associated with a history of STI (white adjusted prevalence ratio [APR]: 1.54, 95\% CI 1.14, 2.06; Black APR: 1.26, 95\% CI 1.02, 1.56); the association between incarceration and PID was null among white women (APR: 0.99 , 95\% CI $0.47,2.09$ ) and elevated among Black women (APR: 2.82, 95\% CI 1.36, 5.83). Prior incarceration did not appear associated with pregnancy loss among white women (APR: 1.01, 95\%CI 0.70, 1.45), but was associated among Black women (APR: 1.38, 95\% CI: 0.97, 1.97), with STI appearing to partially mediate.

Conclusion Pregnancy loss may be elevated among Black women who have been incarcerated, and incarceration-related increases in STI may account for some of this association. Sexual and reproductive health care providers may want to assess history of incarceration among patients and, given the stronger associations among Black women and the higher prevalence of incarceration in this group, incarceration may have particularly deleterious consequences for their reproductive health, underscoring the need to consider alternatives to incarceration to address racial inequities.

\section{P124 SUBSTANCE ABUSE, VIOLENCE, AND SEXUAL RISK AMONG ADOLESCENT CIS-GENDER WOMEN WHO ARE AT HIGH-RISK FOR HUMAN IMMUNODEFICIENCY VIRUS}

${ }^{1} \mathrm{G}$ Vavala*, ${ }^{1} \mathrm{Q}$ Wang, ${ }^{1} \mathrm{C}$ Stafylis, ${ }^{2} \mathrm{~S}$ Jimenez, ${ }^{2} \mathrm{~W}$ Ramos, ${ }^{3} \mathrm{M}$ Ocasio, ${ }^{2} \mathrm{~A}$ Romero-Espinoza, ${ }^{4} \mathrm{R}$ Flynn, ${ }^{5} \mathrm{M}$ Fernandez, ${ }^{2} \mathrm{D}$ Swendeman, ${ }^{2} \mathrm{~W}$ Comulada, ${ }^{1} \mathrm{~J}$ Klausner. ${ }^{1}$ Division of Infectious Diseases, David Geffen School of Medicine, University of California Los Angeles, Los Angeles, USA; ${ }^{2}$ Department of Psychiatry and Biobehavioral Sciences, David Geffen School of Medicine, University of California Los Angeles, Los Angeles, USA; ${ }^{3}$ Department of Pediatrics/Section of Adolescent Medicine, Tulane University School of Medicine, New Orleans, USA; ${ }^{4}$ Los Angeles LGBT Center, Los Angeles, USA; ${ }^{5}$ Nova Southeastern University, Fort Lauderdale, USA

\subsection{6/sextrans-2021-sti.241}

Background The substance abuse, violence, and AIDS (SAVA) syndemic framework offers a holistic approach to analyze these mutually reinforcing epidemics among women with or at-risk for HIV/AIDS. We investigated the relationship between SAVA conditions and sexual risk behavior among adolescent cis-gender women.

Methods We recruited high-risk adolescents into a large prospective Adolescent Trials Network Study between May 2017 2018 in Los Angeles and New Orleans. Participants selfreported risky sexual behaviors in the past four months including condomless sex, number of sex partners, and substance use before sex. We categorized participants into having 0-3 SAVA condition(s) based on the presence or absence of self-reported substance abuse in the past 4 months, including marijuana, crack, heroine, ecstasy, and methamphetamine, childhood sexual abuse (CSA), and intimate partner violence (IPV) at baseline. We used logistic regression models to examine the association between the number of SAVA conditions and sexual risk behavior.

Results Our study included 204 cis-gender women, median age of 20 years (IQR 19-22). Overall, 80.4\% reported recent substance use, $24.5 \%$ reported recent IPV, and 39.7\% reported
CSA. Among all participants, 47.6\%, 32.4\%, and $10.8 \%$ reported occurrence of one, two, and all three SAVA condition(s), respectively. The majority of participants $(77.5 \%)$ reported condomless sex, $42.7 \%$ had more than one sex partner, and $36.8 \%$ reported substance use before sex. Each additional SAVA condition experienced by women was associated with having more than one sex partners (adjusted OR = $1.67 ; 95 \%$ confidence interval $[\mathrm{CI}]=1.17,2.40)$ and substance use before sex $(\mathrm{OR}=1.41 ; 95 \% \mathrm{CI}=0.99,2.02)$.

Conclusions In our study SAVA conditions were common. We found an association between the increased number of SAVA conditions and sexual risk behavior. How to mitigate the impact of SAVA conditions on sexual risk behavior needs further study.

\section{P125 BIOLOGICAL OUTCOME MEASUREMENT THROUGH MAIL-BASED TESTING FOR HIV, STIS, AND SUBSTANCE USE AMONG ADOLESCENTS}

${ }^{1} \mathrm{G}$ Vavala*, ${ }^{1} \mathrm{Q}$ Wang, ${ }^{1} \mathrm{C}$ Stafylis, ${ }^{2} \mathrm{~W}$ Ramos, ${ }^{3} \mathrm{M}$ Ocasio, ${ }^{2} \mathrm{~A}$ Romero-Espinoza, ${ }^{4} \mathrm{R}$ Flynn, ${ }^{5} \mathrm{M}$ Fernandez, ${ }^{2} \mathrm{D}$ Swendeman, ${ }^{2} \mathrm{~W}$ Comulada, ${ }^{1} \mathrm{~J}$ Klausner, the Adolescent Medicine Trials Network (ATN) CARES Team. 'Division of Infectious Diseases, David Geffen School of Medicine, University of California Los Angeles, Los Angeles, USA; ${ }^{2}$ Department of Psychiatry and Biobehavioral Sciences, David Geffen School of Medicine, University of California Los Angeles, Los Angeles, USA; ${ }^{3}$ Department of Pediatrics/Section of Adolescent Medicine, Tulane University School of Medicine, New Orleans, USA; ${ }^{4}$ Los Angeles LGBT Center, Los Angeles, USA; ${ }^{5}$ Nova Southeastern University, Fort Lauderdale, USA

\subsection{6/sextrans-2021-sti.242}

Background COVID-19 disrupted in-person clinical research activities. In response, the Adolescent Medicine Trials Network (ATN) CARES study instituted mail-based testing for human immunodeficiency virus (HIV), sexually transmitted infections (STIs), and substance use screening. We assessed the acceptability and feasibility of the ATN CARES mail-based testing program.

Methods From September 2020- December 2020, trained study staff sent myLAB Box $\odot$ mail-based test kits and written instructions to gay, bisexual, and transgender study participants between the ages of 16-26 years. The test kits included a dried blood spot collection card for HIV (4th generation antigen/antibody EIA) and syphilis (TP-EIA) testing, swabs and a urine collection kit for the detection of extragenital and genital Chlamydia trachomatis and Neisseria gonorrhoeae infection, and a urine collection kit for the screening of six substances. Staff tracked shipment status, and when delivery was identified, contacted participants to assist them with selfcollection and test kit return.

Results Of the 65 youth who participated to date, 49.2\% (32/ $65)$ returned their kit and completed at least one test. Those 32 participants had a median age of 23.5 years (IQR 22-25). The median number of days from test kit ordered to results was 18.5 days (IQR 15-23.5); median number of days from collection to results was 10 days (IQR 8.5-12). MyLAB Box ( reported a total of 219 test

Results 30 HIV, 22 syphilis, 56 chlamydia, 56 gonorrhea, and 55 drug tests processed. Of the 219 tests, $7.3 \%$ (16) were rejected due to specimen inadequacy (6 dried blood spot cards, 2 anal swabs, 8 urine specimens).

Conclusions Mail-based testing is a promising way to monitor a variety of biological study outcomes among adolescent study participants. Future studies should further investigate how to strengthen utilization and fidelity of mail-based testing. 